\title{
Association between health literacy and metabolic syndrome or healthy lifestyle characteristics among community-dwelling Japanese people
}

\author{
Hirohide Yokokawa ${ }^{1 *}$, Hiroshi Fukuda ${ }^{1}$, Motoyuki Yuasa² ${ }^{2}$ Hironobu Sanada ${ }^{3,4}$, Teruhiko Hisaoka ${ }^{1}$
} and Toshio Naito ${ }^{1}$

\begin{abstract}
Background: Few studies have assessed the association between health literacy $(\mathrm{HL})$ and healthy lifestyle characteristics among Japanese people, and reports on the association between $\mathrm{HL}$ and prevalence of metabolic syndrome are also scarce.

Methods: The present cross-sectional study included 1817 (781 men and 1036 women) Japanese individuals who participated in a medical health checkup at Bange Kosei General Hospital and Takada Kosei Hospital in Fukushima, Japan, from April 2013-2014. Information regarding HL and healthy lifestyle characteristics listed in Breslow's seven health practices was collected by self-administered questionnaire.

Results: In multivariate logistic analysis, higher $\mathrm{HL}(\geq 14)$ was positively associated with healthy lifestyle characteristics [odds ratio $(\mathrm{OR})=2.08,95 \%$ confidence interval $(\mathrm{Cl})=1.33-3.23$ ] and inversely associated with prevalence of metabolic syndrome $(\mathrm{OR}=0.67,95 \% \mathrm{Cl}=0.48-0.95)$ among men. Among $\mathrm{HL}$ items, the ability to make decisions based on health-related information was significantly associated with healthy lifestyle characteristics (OR $=2.04,95 \%$ $\mathrm{Cl}=1.34-3.10$ for men, $\mathrm{OR}=1.38,95 \% \mathrm{Cl}=1.30-1.85$ for women) and inversely associated with prevalence of metabolic syndrome $(\mathrm{OR}=0.62,95 \% \mathrm{Cl}=0.44-0.88$ for men, $\mathrm{OR}=0.68,95 \% \mathrm{Cl}=0.49-0.95$ for women $)$ in both sexes.
\end{abstract}

Conclusions: We found positive associations between $\mathrm{HL}$ and healthy lifestyle characteristics and an inverse association with prevalence of metabolic syndrome among men. Our findings also suggest that men and women are likely to engage in health-promoting behaviors and make decisions based on health-related information. These findings highlight the importance of comprehensive assessments, including $\mathrm{HL}$, for health promotion in the community.

Keywords: Epidemiology, Health Promotion, Health literacy, Metabolic syndrome, Lifestyle

\section{Background}

In recent decades, both developed and developing countries have faced a dramatic increase in the number of adults suffering from non-communicable diseases (NCDs) such as diabetes, cardiovascular disease (CVD), and chronic kidney disease (CKD) [1]. Metabolic

\footnotetext{
*Correspondence: yokokawa@pa3.so-net.ne.jp

1 Department of General Medicine, Juntendo University School

of Medicine, Hongo 2-1-1, Bunkyo-ku, Tokyo 113-8421, Japan

Full list of author information is available at the end of the article
}

syndrome, in particular, has received considerable attention [2-4]. Metabolic syndrome is a concurrence of disturbed glucose and insulin metabolism, abdominal fat distribution, mild dyslipidemia, and hypertension [4], and is an important clinical condition given its association with the subsequent development of type 2 diabetes mellitus and cardiovascular disease [2-4]. The prevalence of metabolic syndrome and suspected individuals is estimated to be 20.1 million in Japan among those aged $\geq 40$ years in 2008 [5]. Metabolic syndrome is known to increase the risk of mortality from coronary 
heart disease (CHD) and cardiovascular disease (CVD), and is also a risk factor for cognitive impairment $[6,7]$. Therefore, modification of lifestyle characteristics at the individual level has been emphasized in the prevention of this syndrome.

Although the growing human and economic costs of metabolic syndrome have been recognized, and action plans to prevent it have been developed, the impact on health outcomes has been limited [5]. Unhealthy lifestyles likely contribute to the development of metabolic syndrome, and the importance of lifestyle modification has been emphasized through advanced communication tools [8].

Health information is important for people to understand and engage in the management of their own health. With an increasing amount of health information available through media reports and the internet, many health information resources can easily be distributed to the general population [9-11]. However, adequate use of these resources depends on an individual's skill in finding and applying the information [12]. These skills have been conceptualized as "health literacy" (HL). Based on the World Health Organization (WHO) definition, a model of HL has been proposed that includes three levels, and assumes both individual and population benefits at each level: functional, communicative, and critical literacy [13]. Individuals with adequate HL are considered likely to adopt healthy lifestyles [14], and a brief questionnaire was developed to easily assess HL [15]. However, only a few studies have estimated the association between $\mathrm{HL}$ and healthy lifestyle characteristics among Japanese people [15-17], and reports on the association between $\mathrm{HL}$ and prevalence of metabolic syndrome are especially limited.

This study aimed to examine sex-specific associations between HL and healthy lifestyle characteristics and prevalence of metabolic syndrome among a Japanese community-dwelling population using a concise, newly developed questionnaire.

\section{Methods}

\section{Participants}

The present cross-sectional study included 1825 Japanese individuals who participated in a medical health checkup at Bange Kosei General Hospital and Takada Kosei Hospital in Fukushima, Japan, from April 2013-2014. Of these, eight were excluded due to refusal to participate or missing data. Thus, a total of 1817 participated in this study.

\section{Variables}

Body height and weight were measured in the standing position. Body mass index (BMI) was calculated based on body weight $(\mathrm{kg})$ divided by height squared $\left(\mathrm{m}^{2}\right)$. Both systolic (SBP) and diastolic blood pressure (DBP) were obtained as the mean of two measurements on the upper arm after the participant had been seated for at least $5 \mathrm{~min}$. We also collected information for the following lipid-related items: total cholesterol (mg/dL; TC), high-density lipoprotein-cholesterol (mg/dL; HDL-C), and triglycerides (mg/dL; TG). Low-density lipoproteincholesterol $(\mathrm{mg} / \mathrm{dL}$; LDL-C) was estimated using the Friedwald equation ([TC]-[HDL-C]-[TG/5]) [18]. We measured fasting plasma glucose (FPG) concentrations by the hexokinase ultraviolet method using an automated analyzer after more than $9 \mathrm{~h}$ of fasting.

We diagnosed metabolic syndrome according to the National Cholesterol Education Program's Adult Treatment Panel III report (ATP III) [19]. For waist circumference, we diagnosed abdominal obesity using Japan-specific criteria (male waist circumference $\geq 90 \mathrm{~cm}$, female waist circumference $\geq 80 \mathrm{~cm}$ ), in accordance with the International Diabetes Federation (IDF) Worldwide Definition of metabolic syndrome [4]. When 3 out of 5 of the following listed characteristics are present, a diagnosis of metabolic syndrome is made: (1) abdominal obesity (male waist circumference $\geq 90 \mathrm{~cm}$, female waist circumference $\geq 80 \mathrm{~cm}$ ), (2) raised TG level $(\geq 150 \mathrm{mg} / \mathrm{dL}$ ) or specific treatment for this lipid abnormality, (3) reduced HDL-C level ( $\leq 40 \mathrm{mg}$ / $\mathrm{dL}$ in men, $\leq 50 \mathrm{mg} / \mathrm{dL}$ in women) or specific treatment for this lipid abnormality, (4) raised blood pressure (systolic $\mathrm{BP} \geq 130 \mathrm{mmHg}$ or diastolic $\mathrm{BP} \geq 85 \mathrm{mmHg}$ ) or treatment for previously diagnosed hypertension, and (5) raised fasting plasma glucose $(\geq 110 \mathrm{mg} / \mathrm{dL})$ or previously diagnosed type 2 diabetes [19].

We interviewed participants regarding their medical histories for atherosclerotic complications (cardiovascular or cerebrovascular diseases) and lifestyle-related disorders (hypertension, dyslipidemia, or diabetes mellitus). We then asked participants to complete self-administrated questionnaires, which included $\mathrm{HL}$ and healthy lifestyle characteristics listed in Breslow's seven health practices, such as alcohol consumption, smoking behavior, exercise frequency, obesity (BMI), sleep duration, breakfast, and snacks between meals [20].

To evaluate $\mathrm{HL}$, a validated questionnaire that included three items for communicative HL (items 1-3) and two items for critical HL (items 4, 5) was used [15]. A previous study using the questionnaire reported Cronbach's $\alpha$ of communicative and critical HL scales to be 0.77 and 0.65 , respectively [15]. Participants could easily complete the questionnaire because it has only five items. These items asked whether the participant would be able to (1) collect health-related information from various sources, (2) extract the relevant information, (3) understand and 
communicate the obtained information, (4) consider the credibility of the information, and (5) make decisions based on the information, specifically in the context of health-related issues. The participant rated each item on a 5-point Likert scale ranging from 1 (strongly disagree) to 5 (strongly agree).

We defined healthy lifestyle characteristics as the following responses based on Breslow's seven health practices [20, 21]: alcohol consumption (non-everyday drinker), smoking behavior (non-current smoker), exercise frequency (twice or more times per week), BMI (18.5-24.9), sleep duration (7-8 h), breakfast (every morning), and snacks between meals (no).

\section{Statistical analysis}

Variables are presented as mean \pm standard deviation (SD) for continuous variables or prevalence (\%) for categorical variables. Total HL score was dichotomized based on the median score (low $\mathrm{HL}<14$, high $\mathrm{HL} \geq 14$ ). We used the $t$ test for continuous variables and the Chi square test or Fisher's exact test for comparisons of proportions between sexes. To estimate the potential of HL to promote a healthy lifestyle with 6-7 healthy characteristics, which are most likely to be present in the healthiest lifestyle [21], logistic regression analysis was performed using the following models. Model 1 was adjusted for age (years), total HL score ( $\geq 14$ vs. $<14$ ), and atherosclerotic complications (cardiovascular and cerebrovascular diseases). Model 2 was adjusted for age (years), five HL item scores ( $\geq 4$ vs. $<4$ ), and atherosclerotic complications (cardiovascular and cerebrovascular diseases).

Logistic regression analysis was then performed to estimate associations between metabolic syndrome and total HL score $(\geq 14$ vs. $<14)$ or five HL item scores ( $\geq 4$ vs. $<4)$ for both sexes. Model 1 was adjusted for age (years), total HL score ( $\geq 14$ vs. $\leq 14$ ), atherosclerotic complications (cardiovascular and cerebrovascular diseases), alcohol consumption (non-everyday drinker), and smoking behavior (non-current smoker). Model 2 was adjusted for age (years), five HL item scores ( $\geq 4$ vs. $\leq 4)$, atherosclerotic complications (cardiovascular and cerebrovascular diseases), alcohol consumption (non-everyday drinker), and smoking behavior (non-current smoker).

All statistical analyses were performed using the Statistical Package for Social Sciences version 22 (IBM SPSS Inc., Chicago, IL, USA). $P<0.05$ was considered statistically significant.

\section{Ethical considerations}

This survey was conducted according to the Ethical Guidelines for Epidemiological Studies established by the Japanese government [22], and the Ethics Committee of Juntendo University approved the research protocol (No. 833). We obtained informed consent from all participants.

\section{Results}

Table 1 summarizes the basic characteristics of male participants stratified by HL level. Among men, mean age was 51.2 years in the lower HL group, and 51.0 years in the higher HL group. TG and FPG were significantly lower in the higher HL group compared to the lower HL group. Although proportions of those with metabolic syndrome, waist circumference $(\geq 90 \mathrm{~cm})$, and raised fasting plasma glucose $(\geq 110 \mathrm{mg} / \mathrm{dL})$ did not significantly differ between higher and lower HL groups, the differences were nearly significant $(P=0.06,0.08$, and 0.05 , respectively). As for healthy lifestyle characteristics, proportions of smoking behavior (non-smoker), BMI (18.5-24.9), and exercise frequency (twice or more per week) were also significantly higher in the higher HL group compared to the lower HL group. In addition, the proportion of participants with 6-7 total healthy lifestyle items was significantly higher in the higher HL group. Among women, mean age was 49.2 years in lower and higher HL groups. There were no significant differences between higher and lower HL groups in any of the basic characteristics (Table 2). The prevalence of metabolic syndrome in all participants, men, and women were 15.6, 20.9 , and $11.6 \%$, respectively.

Table 3 show multivariate regression analyses for associations between HL and 6-7 healthy lifestyle characteristics. Among men, a higher HL (total scale score $\geq 14$ ) was significantly associated with having 6-7 healthy lifestyle characteristics in Model 1 [odds ratio $(\mathrm{OR})=2.08$, $95 \%$ confidence interval $(\mathrm{CI})=1.33-3.23)]$. In addition, "Understanding and communicating the information" and "Making decisions based on the (health-related) information" were significantly associated with having 6-7 healthy lifestyle characteristics in Model $2[(\mathrm{OR}=1.63$, $95 \% \mathrm{CI}=1.08-2.47),(\mathrm{OR}=2.04,95 \% \mathrm{CI}=1.34-3.10)]$ (Table 3). Among women, no association was observed between HL and healthy lifestyle characteristics (Table 3).

As for metabolic syndrome, a higher HL (total scale score $\geq 14$ ) was significantly associated with a lower prevalence of metabolic syndrome in Model $1(\mathrm{OR}=0.67$, $95 \%$ CI $=0.48-0.95$ ) among men (Table 4). "Making decisions based on the (health-related) information" was significantly associated with a lower prevalence of metabolic syndrome in Model 2 (OR $=0.62,95 \%$ $\mathrm{CI}=0.44-0.88$ for men, $\mathrm{OR}=0.68,95 \% \mathrm{CI}=0.49-0.95$ for women) in both sexes (Table 4).

\section{Discussion}

Our analysis of cross-sectional data showed that higher HL was significantly associated with healthy lifestyle 
Table 1 Health literacy specific characteristics among men $(\mathbf{N}=781)$

\begin{tabular}{|c|c|c|c|}
\hline & \multicolumn{2}{|l|}{ Mean $( \pm S D)$ or $\mathrm{N}(\%)$} & \multirow[t]{2}{*}{$P^{a}$} \\
\hline & $\begin{array}{l}\text { Low health } \\
\text { literacy }(\leq 13)(N=350)\end{array}$ & $\begin{array}{l}\text { High health } \\
\text { literacy }(\geq 14)(N=431)\end{array}$ & \\
\hline Age (years) & $51.2(9.9)$ & $51.0(9.9)$ & \\
\hline \multicolumn{4}{|l|}{ Anthropometric measurements } \\
\hline Height (cm) & $170.0(6.5)$ & $170.2(6.4)$ & \\
\hline Body weight (kg) & $68.6(12.1)$ & $68.8(11.1)$ & \\
\hline Body mass index (BMI) & $23.7(3.8)$ & $23.7(3.4)$ & \\
\hline Waist circumference (cm) & $85.0(9.5)$ & $84.6(8.8)$ & \\
\hline \multicolumn{4}{|l|}{ Atherosclerotic complications } \\
\hline Cardiovascular disease & $13(3.7)$ & $18(4.2)$ & \\
\hline Cerebrovascular disease & $3(0.9)$ & $8(1.9)$ & \\
\hline \multicolumn{4}{|l|}{ Hypertension-related factors } \\
\hline Systolic blood pressure (mmHg) & $133.5(19.4)$ & $135.0(17.7)$ & \\
\hline Diastolic blood pressure $(\mathrm{mmHg})$ & $81.1(12.4)$ & $81.7(12.1)$ & \\
\hline Antihypertensive drug use (yes) & $72(20.6)$ & $102(23.7)$ & \\
\hline \multicolumn{4}{|l|}{ Lipid-related items } \\
\hline High-density lipoprotein cholesterol (mg/dL) & $57.1(15.2)$ & $58.9(15.5)$ & \\
\hline Low-density lipoprotein cholesterol (mg/dL) & $130.1(32.7)$ & $128.2(33.0)$ & \\
\hline Triglycerides (mg/dL) & $168.1(132.5)$ & $149.4(106.4)$ & * \\
\hline Antidyslipidemic drug use (yes) & $29(8.3)$ & $41(9.5)$ & \\
\hline \multicolumn{4}{|l|}{ Diabetes-related items } \\
\hline Fasting plasma glucose (mg/dL) & $105.1(27.1)$ & $101.3(18.4)$ & * \\
\hline Antidiabetic drug use (yes) & $12(3.4)$ & $17(3.9)$ & \\
\hline Metabolic syndrome (yes) & $80(24.0)$ & $75(18.3)$ & $\mathrm{b}$ \\
\hline Waist circumference $(\geq 90 \mathrm{~cm})$ & $107(32.0)$ & $107(26.2)$ & $\mathrm{b}$ \\
\hline Raised triglyceride ( $\geq 150 \mathrm{mg} / \mathrm{dL})$ & $132(37.7)$ & $149(34.6)$ & \\
\hline Reduced high density lipoprotein cholesterol ( $\leq 40$ mg/dL) & $25(7.1)$ & $26(6.0)$ & \\
\hline Raised blood pressure (systolic $\geq 130 \mathrm{mmHg}$ or diastolic $\geq 85 \mathrm{mmHg}$ ) & $228(65.3)$ & $301(69.8)$ & \\
\hline Raised fasting plasma glucose ( $\geq 110 \mathrm{mg} / \mathrm{dL})$ & $83(23.7)$ & $78(18.1)$ & $\mathrm{b}$ \\
\hline \multicolumn{4}{|l|}{ Healthy lifestyle characteristics } \\
\hline Alcohol consumption (non-everyday drinker) & $161(46.0)$ & $223(51.7)$ & \\
\hline Smoking behavior (non-current smoker) & $174(49.7)$ & $262(60.8)$ & $* *$ \\
\hline Exercise frequency (2 times or more per week) & $48(13.7)$ & $96(22.3)$ & $* *$ \\
\hline Body mass index (18.5-24.9) & $216(61.7)$ & $293(68.0)$ & $*$ \\
\hline Sleep hours (6-9) & $220(62.9)$ & $290(67.3)$ & \\
\hline Breakfast (every morning) & $277(79.1)$ & $355(82.4)$ & \\
\hline Snack between meals (no) & $287(82.0)$ & $371(86.1)$ & \\
\hline Total number of healthy lifestyle items & $4.0(1.2)$ & $4.4(1.2)$ & $* *$ \\
\hline $\begin{array}{l}\text { Proportion of participants with } 6 \text { or } 7 \text { total number of healthy lifestyle } \\
\text { items }\end{array}$ & $34(9.7)$ & $76(17.6)$ & $* *$ \\
\hline \multicolumn{4}{|l|}{ Health literacy } \\
\hline Seeking information from various sources & $3.3(1.0)$ & $4.1(0.6)$ & $* *$ \\
\hline Extracting relevant information & $3.0(0.9)$ & $4.0(0.5)$ & $* *$ \\
\hline Understanding and communicating the information & $2.7(0.7)$ & $3.9(0.5)$ & $* *$ \\
\hline Considering the credibility of the information & $2.7(0.7)$ & $3.8(0.5)$ & ** \\
\hline Making decisions based on the information & $2.8(0.8)$ & $3.7(0.6)$ & ** \\
\hline Total scale score & $11.1(2.1)$ & $15.5(1.3)$ & ** \\
\hline
\end{tabular}

a $* * P<0.01, * P<0.05$

${ }^{\mathrm{b}}$ Borderline significance $0.05<P<0.10$ 
Table 2 Health literacy specific characteristics among women $(N=1036)$

\begin{tabular}{|c|c|c|c|}
\hline & \multicolumn{2}{|l|}{ Mean ( \pm SD) or N (\%) } & \multirow[t]{2}{*}{$P^{\mathrm{a}}$} \\
\hline & $\begin{array}{l}\text { Low health literacy } \\
(<14)(\mathrm{N}=437)\end{array}$ & $\begin{array}{l}\text { High health literacy } \\
(\geq 14)(N=599)\end{array}$ & \\
\hline Age (years) & $49.2(9.7)$ & $49.2(9.5)$ & \\
\hline \multicolumn{4}{|l|}{ Anthropometric measurements } \\
\hline Height (cm) & $157.4(6.3)$ & $157.4(5.8)$ & \\
\hline Body weight (kg) & $55.5(9.8)$ & $55.1(9.3)$ & \\
\hline Body mass index (BMI) & $22.4(3.9)$ & $22.3(3.7)$ & \\
\hline Waist circumference (cm) & $80.2(9.8)$ & $80.1(9.3)$ & \\
\hline \multicolumn{4}{|l|}{ Atherosclerotic complications } \\
\hline Cardiovascular disease (yes) & $3(0.7)$ & $9(1.5)$ & \\
\hline Cerebrovascular disease (yes) & $5(1.1)$ & $4(0.7)$ & \\
\hline \multicolumn{4}{|l|}{ Hypertension-related factors } \\
\hline Systolic blood pressure $(\mathrm{mmHg})$ & $124.7(18.4)$ & $124.9(18.8)$ & \\
\hline Diastolic blood pressure (mmHg) & $76.1(11.1)$ & $76.0(11.4)$ & \\
\hline Antihypertensive drug use (yes) & $58(13.3)$ & $69(11.5)$ & \\
\hline \multicolumn{4}{|l|}{ Lipid-related items } \\
\hline High-density lipoprotein cholesterol (mg/dL) & $71.3(17.5)$ & $70.9(16.2)$ & \\
\hline Low-density lipoprotein cholesterol (mg/dL) & $122.4(32.0)$ & $123.7(31.4)$ & \\
\hline Triglycerides (mg/dL) & $92.7(53.6)$ & $92.4(57.3)$ & \\
\hline Antidyslipidemic drug use (yes) & $42(9.6)$ & $52(8.7)$ & \\
\hline \multicolumn{4}{|l|}{ Diabetes-related items } \\
\hline Fasting plasma glucose (mg/dL) & $94.3(17.3)$ & $93.8(17.0)$ & \\
\hline Antidiabetic drug use (yes) & $8(1.8)$ & $10(1.7)$ & \\
\hline Metabolic syndrome (yes) & $53(12.5)$ & $64(11.0)$ & \\
\hline Waist circumference $(\geq 80 \mathrm{~cm})$ & $216(50.9)$ & $285(49.0)$ & \\
\hline Raised triglyceride ( $\geq 150 \mathrm{mg} / \mathrm{dL}$ ) & $50(11.4)$ & $60(10.0)$ & \\
\hline Reduced high density lipoprotein cholesterol ( $\leq 40$ mg/dL) & $29(6.6)$ & $43(7.2)$ & \\
\hline Raised blood pressure (systolic $\geq 130 \mathrm{mmHg}$ or diastolic $\geq 85 \mathrm{mmHg}$ ) & $204(46.7)$ & $252(42.1)$ & \\
\hline Raised fasting plasma glucose ( $\geq 110 \mathrm{mg} / \mathrm{dL})$ & $33(7.6)$ & $34(5.7)$ & \\
\hline \multicolumn{4}{|l|}{ Healthy lifestyle characteristics } \\
\hline Alcohol consumption (non-everyday drinker) & $361(82.6)$ & $509(85.0)$ & \\
\hline Smoking behavior (non-current smoker) & $371(84.9)$ & $516(86.1)$ & \\
\hline Exercise frequency (2 times or more per week) & $32(7.3)$ & $58(9.7)$ & \\
\hline Body mass index (18.5-24.9) & $307(70.3)$ & $406(67.8)$ & \\
\hline Sleep hours (6-9) & $234(53.5)$ & $323(53.9)$ & \\
\hline Breakfast (every morning) & $365(83.5)$ & $512(85.5)$ & \\
\hline Snack between meals (no) & $329(75.3)$ & $446(74.5)$ & \\
\hline Total number of healthy lifestyle items & $4.6(1.2)$ & $4.6(1.2)$ & \\
\hline $\begin{array}{l}\text { Proportion of participants with } 6 \text { or } 7 \text { total number of healthy lifestyle } \\
\text { items }\end{array}$ & $97(22.2)$ & $148(24.7)$ & \\
\hline \multicolumn{4}{|l|}{ Health literacy } \\
\hline Seeking information from various sources & $3.5(0.9)$ & $4.2(0.6)$ & ** \\
\hline Extracting relevant information & $3.1(0.9)$ & $4.1(0.5)$ & ** \\
\hline Understanding and communicating the information & $2.7(0.7)$ & $3.9(0.5)$ & ** \\
\hline Considering the credibility of the information & $2.6(0.7)$ & $3.7(0.6)$ & ** \\
\hline Making decisions based on the information & $2.8(0.7)$ & $3.8(0.6)$ & ** \\
\hline Total scale score & $11.2(2.0)$ & $15.5(1.3)$ & ** \\
\hline
\end{tabular}

a $* * P<0.01, * P<0.05$ 
Table 3 Logistic regression analysis of health literacy for men and women with 6-7 healthy lifestyle characteristics

\begin{tabular}{|c|c|c|c|c|c|c|c|c|c|}
\hline & \multirow{2}{*}{\multicolumn{3}{|c|}{ Univariate analysis }} & \multicolumn{6}{|c|}{ Multivariate analysis } \\
\hline & & & & \multicolumn{3}{|c|}{ Model $1^{c}$} & \multicolumn{3}{|c|}{ Model $2^{d}$} \\
\hline & $\mathrm{OR}^{\mathrm{a}}$ & $95 \% \mathrm{Cl}^{\mathrm{b}}$ & $P^{\mathrm{e}}$ & $\mathrm{OR}^{\mathrm{a}}$ & $95 \% \mathrm{Cl}^{\mathrm{b}}$ & $P^{e}$ & $\mathrm{OR}^{\mathrm{a}}$ & $95 \% \mathrm{Cl}^{\mathrm{b}}$ & $\mathbf{P}^{\mathrm{e}}$ \\
\hline \multicolumn{10}{|l|}{ For men $(\mathrm{N}=781)$} \\
\hline \multicolumn{10}{|l|}{ Health literacy } \\
\hline Seeking information from various sources ( $\geq 4$ vs. $<4)$ & 1.26 & $0.78-2.02$ & & - & - & & 1.44 & $0.89-2.34$ & \\
\hline Extracting relevant information ( $\geq 4$ vs. $<4$ ) & 1.31 & $0.85-2.01$ & & - & - & & 1.50 & $0.97-2.32$ & \\
\hline Understanding and communicating the information ( $\geq 4$ vs. $<4)$ & 1.59 & $1.06-2.39$ & ** & - & - & & 1.63 & $1.08-2.47$ & * \\
\hline Considering the credibility of the information ( $\geq 4$ vs. $<4$ ) & 1.49 & $0.99-2.23$ & & - & - & & 1.39 & $0.92-2.10$ & \\
\hline Making decisions based on the information ( $\geq 4$ vs. <4) & 2.16 & $1.42-3.27$ & ** & - & - & & 2.04 & $1.34-3.10$ & $* *$ \\
\hline Total score ( $\geq 14$ vs. <14) & 1.99 & $1.29-3.06$ & ** & 2.08 & $1.33-3.23$ & ** & - & - & \\
\hline \multicolumn{10}{|l|}{ For women $(\mathrm{N}=1036)$} \\
\hline \multicolumn{10}{|l|}{ Health literacy } \\
\hline Seeking information from various sources ( $\geq 4$ vs. $<4$ ) & 0.87 & $0.61-1.23$ & & - & - & & 1.05 & $0.73-1.51$ & \\
\hline Extracting relevant information ( $\geq 4$ vs. $<4)$ & 0.81 & $0.60-1.09$ & & - & - & & 0.87 & $0.64-1.18$ & \\
\hline Understanding and communicating the information ( $\geq 4$ vs. $<4$ ) & 1.07 & $0.80-1.42$ & & - & - & & 0.98 & $0.73-1.31$ & \\
\hline Considering the credibility of the information ( $\geq 4$ vs. $<4$ ) & 1.56 & $1.17-2.01$ & ** & - & - & & 1.43 & $1.07-1.92$ & * \\
\hline Making decisions based on the information ( $\geq 4$ vs. $<4$ ) & 1.54 & $1.15-2.05$ & ** & - & - & & 1.38 & $1.30-1.85$ & $* *$ \\
\hline Total score $(\geq 14$ vs. <14) & 1.15 & $0.86-1.54$ & & 1.17 & $0.86-1.58$ & & - & - & \\
\hline \multicolumn{10}{|l|}{ a Odds ratio } \\
\hline \multicolumn{10}{|l|}{ b $95 \%$ confidence interval } \\
\hline \multicolumn{10}{|c|}{ c Model 1 was adjusted for age (years), total health literacy score ( $\geq 14$ vs. $<14$ ), and atherosclerotic complications (cardiovascular and cerebrovascular diseases) } \\
\hline \multicolumn{10}{|c|}{ d Model 2 was adjusted for age (years), five health literacy items ( $\geq 4$ vs. $<4$ ), and atherosclerotic complications (cardiovascular and cerebrovascular diseases) } \\
\hline e $* * P<0.01, * P<0.05$ & & & & & & & & & \\
\hline
\end{tabular}

characteristics, specifically non-smoker status and high exercise frequency, among men. Moreover, the proportion of metabolic syndrome was lower and the proportion of ideal BMI (18.5-24.9) was higher with higher HL compared with lower HL among men. Multivariate analyses revealed that higher HL $(\geq 14)$ was significantly associated with having 6-7 healthy lifestyle characteristics, and was also inversely associated with metabolic syndrome among men, whereas no significant association was found among women. The ability to make decisions based on health-related information was positively associated with a healthy lifestyle and inversely associated with metabolic syndrome in both sexes. Similar to our results, median scores of communicative and critical HL in a previous study that used the same scales were both 4 [13]. Thus, our study population may be similar to the general Japanese population. To the best of our knowledge, this study is the first to examine associations between HL and healthy lifestyle characteristics and prevalence of metabolic syndrome among Japanese people. Our results highlight the importance of improving HL to prevent metabolic syndrome through a healthy lifestyle.
Only a few studies have examined the association between HL and obesity, between HL and metabolic syndrome, and between HL and BMI among adult individuals $[20,21]$. A cross-sectional study, which was conducted in the United States using the Newest Vital Sign (NVS) to measure HL, reported that BMI predicted adequate $\mathrm{HL}$ among adults $(\mathrm{OR}=1.040,95 \% \mathrm{CI}=1.016-$ 1.065) [23]. In another cross-sectional survey, which examined the association between HL measured by NVS and BMI among 364 native Hawaiian and Pacific Islanders in the United States, low NVS scores were associated with increased BMI $(r=-0.12, \mathrm{P}=0.027)[24]$. Associations between HL and child and adolescent obesity have also been reported $[25,26]$. In one cross-sectional study that tested the association between child and parental HL (using NVS) and their obesity, children's obesity decreased with higher parental NVS (OR $=0.75$, $95 \% \mathrm{CI}=0.59-1.00)$, and increased with parental obesity $(\mathrm{OR}=2.53,95 \% \mathrm{CI}=1.08-5.94)$ [25]. In a survey that examined the association between child HL using the Short Test of Functional Health Literacy in Adults (STOFHLA) and BMI among 171 overweight children in the United States, child STOFHLA correlated negatively 
Table 4 Logistic regression analysis of health literacy for men and women with metabolic syndrome

\begin{tabular}{|c|c|c|c|c|c|c|c|c|}
\hline & \multirow{2}{*}{\multicolumn{2}{|c|}{ Univariate analysis }} & \multicolumn{6}{|c|}{ Multivariate analysis } \\
\hline & & & \multicolumn{3}{|c|}{ Model $1^{c}$} & \multicolumn{3}{|c|}{ Model $2^{d}$} \\
\hline & $\mathrm{OR}^{\mathrm{a}}$ & $95 \% \mathrm{Cl}^{\mathrm{b}}$ & $\mathrm{OR}^{\mathrm{a}}$ & $95 \% \mathrm{Cl}^{\mathrm{b}}$ & $P^{e}$ & $\mathrm{OR}^{\mathrm{a}}$ & $95 \% \mathrm{Cl}^{\mathrm{b}}$ & $\mathbf{P}^{\mathrm{e}}$ \\
\hline \multicolumn{9}{|l|}{ For men $(\mathrm{N}=742)$} \\
\hline \multicolumn{9}{|l|}{ Health literacy } \\
\hline Seeking information from various sources ( $\geq 4$ vs. <4) & 0.83 & $0.56-1.21$ & - & - & & 0.75 & $0.52-1.09$ & \\
\hline Extracting relevant information ( $\geq 4$ vs. $<4$ ) & 0.82 & $0.58-1.18$ & - & - & & 0.80 & $0.55-1.11$ & \\
\hline Understanding and communicating the information ( $\geq 4$ vs. $<4)$ & 0.80 & $0.57-1.14$ & - & - & & 0.80 & $0.57-1.13$ & \\
\hline Considering the credibility of the information ( $\geq 4$ vs. $<4$ ) & 0.82 & $0.58-1.16$ & - & - & & 0.80 & $0.55-1.10$ & \\
\hline Making decisions based on the information ( $\geq 4$ vs. <4) & 0.64 & $0.45-0.91^{*}$ & - & - & & 0.62 & $0.44-0.88$ & $* *$ \\
\hline Total score ( $\geq 14$ vs. $<14)$ & 0.69 & $0.49-0.97^{*}$ & 0.67 & $0.48-0.95$ & $* *$ & - & - & \\
\hline \multicolumn{9}{|l|}{ For women $(\mathrm{N}=1006)$} \\
\hline \multicolumn{9}{|l|}{ Health literacy } \\
\hline Seeking information from various sources ( $\geq 4$ vs. <4) & 0.62 & $0.43-0.89^{* *}$ & - & - & & 0.81 & $0.55-1.19$ & \\
\hline Extracting relevant information ( $\geq 4$ vs. $<4)$ & 0.67 & $0.48-0.93^{*}$ & - & - & & 0.81 & $0.57-1.13$ & \\
\hline Understanding and communicating the information ( $\geq 4$ vs. $<4)$ & 0.88 & $0.64-1.21$ & - & - & & 0.85 & $0.61-1.18$ & \\
\hline Considering the credibility of the information ( $\geq 4$ vs. $<4$ ) & 0.97 & $0.70-1.33$ & - & - & & 0.89 & $0.64-1.24$ & \\
\hline Making decisions based on the information ( $\geq 4$ vs. $<4$ ) & 0.80 & $0.59-1.10$ & - & - & & 0.68 & $0.49-0.95$ & $*$ \\
\hline Total score $(\geq 14$ vs. $<14)$ & 0.82 & $0.60-1.13$ & 0.80 & $0.57-1.12$ & & - & - & \\
\hline \multicolumn{9}{|l|}{ a Odds ratio } \\
\hline \multicolumn{9}{|l|}{ b $95 \%$ confidence interval } \\
\hline \multicolumn{9}{|c|}{$\begin{array}{l}\text { c Model } 1 \text { was adjusted for age (years), total health literacy score ( } \geq 14 \text { vs. } \leq 14 \text { ), atherosclerotic complications (cardiovascular and cerebrovascular diseases), alcohol } \\
\text { consumption (non-everyday drinker), and smoking behavior (non-current smoker) }\end{array}$} \\
\hline \multicolumn{9}{|c|}{$\begin{array}{l}\text { d Model } 2 \text { was adjusted for age (years), five health literacy items ( } \geq 4 \text { vs. } \leq 4) \text {, atherosclerotic complications (cardiovascular and cerebrovascular diseases), alcohol } \\
\text { consumption (non-everyday drinker), and smoking behavior (non-current smoker) }\end{array}$} \\
\hline
\end{tabular}

with BMI Z score $(r=-0.37, \mathrm{P}<0.01)$ [26]. In this study, we also found that the proportion of ideal BMI (18.524.9) was higher in the higher HL group than in the lower HL group among men. This suggests that a higher HL may contribute to maintaining an ideal body weight. Thus, estimation of HL level may be useful and even necessary for lifestyle modification strategies.

We also found an association between HL level and prevalence of metabolic syndrome among men. Metabolic syndrome represents clusters of cardiovascular risk factors, assuming that cardiovascular risk is amplified more than is expected from the effect of single risk factors $[2-4,27]$. The risk of cardiovascular events and death was found to be markedly greater for individuals with metabolic syndrome compared to those without, regardless of the definition of metabolic syndrome [27]. Thus, preventing metabolic syndrome will be important for the prevention of cardiovascular disease. To date, only a few studies have assessed the association between metabolic syndrome and HL level, and correlations with chronic disease-related HL [28]. Additional studies will be needed to further explore these associations and correlations.
We also found an association between HL level and healthy lifestyle characteristics among men. In a previous Japanese cross-sectional study, which examined the relationship between HL assessed by the 14-item Health Literacy Scale (HLS-14) and health information access, health behavior, and health status, HL was reported to be associated with health information access and health behavior [29]. The authors emphasized the importance of raising HL levels of the target population for health promotion intervention [29]. In another study, which examined the mechanisms linking HL to physical activity and self-reported health among 330 patients with hypertension, significant paths were found from HL to knowledge $(\mathrm{r}=0.22, \mathrm{P}<0.001)$, knowledge to self-efficacy $(\mathrm{r}=0.13, \mathrm{P}<0.01)$, self-efficacy to physical activity $(\mathrm{r}=0.17, \mathrm{P}<0.01)$, and physical activity to health status $(r=0.17, P<0.01)$ [30]. Thus, a higher HL may be related with healthier lifestyle characteristics, which in turn can contribute to a lower prevalence of metabolic syndrome among men.

Among the five assessed items in this study, "Making decisions based on the (health-related) information" was 
significantly associated with metabolic syndrome and healthy lifestyle characteristics in men. The National Panel of people with Chronic illness or Disability (NPCD) in the Netherlands reported that communicative and critical HL are related to some aspects of self-management [31]. Consistent with this, a cross-sectional study in Japanese primary care showed that communicative and critical HL were positively associated with an understanding of diabetes care $(\beta=0.558,0.451, \mathrm{P}<0.001)$ and self-efficacy $(\beta=0.365,0.369, \mathrm{P}<0.001)$, respectively [32]. Thus, critical HL may be related with metabolic syndrome as well as healthy lifestyle characteristics through self-management ability.

We did not find an association between HL level and metabolic syndrome among women in the present study. One potential explanation is that women are likely to have healthier lifestyle characteristics, especially in terms of alcohol consumption and smoking, compared to men. Moreover, the prevalence of metabolic syndrome is lower in women than in men. These differences may explain the lack of association. Further studies will be needed to confirm this.

This study has several limitations. First, there may have been selection bias, as study participants were recruited from those who participated in a health checkup program at two Japanese hospitals. Thus, these participants may have had a higher health awareness compared to non-participants. Large-scale studies that include several regions in Japan and also include individuals who have not received health checkups will be needed in the future. Second, HL and lifestyle characteristics were measured based on self-reported questionnaires. It is possible that these participants may have reported better $\mathrm{HL}$ and healthier lifestyles than is actually true, thereby resulting in an over-estimation of HL and health characteristics. Third, given the cross-sectional design, we could not determine whether there was a causal relationship between HL and healthy lifestyle characteristics. A longitudinal study will be needed to address this issue. Fourth, while the information collected from participants contained a comprehensive set of clinical variables, it cannot be denied that some important factors, especially educational status, were not measured.

\section{Conclusions}

Our study revealed a positive association between HL and healthy lifestyle characteristics and an inverse association with prevalence of metabolic syndrome among men. Our findings also suggest that men and women are likely to engage in health-promoting behaviors and make decisions based on health-related information. These findings highlight the importance of comprehensive assessments, including HL, for health promotion in the community, and the need to improve HL in order to prevent metabolic syndrome through a healthy lifestyle.

\section{Authors' contributions}

$\mathrm{HY}, \mathrm{HF}, \mathrm{MY}, \mathrm{HS}, \mathrm{TH}$, and TN participated in the design of the study. $\mathrm{HY}$ and $\mathrm{HS}$ participated in data collection, analysis of the data, and drafting of the manuscript. HY, HF, and MY conceived of the study, participated in its design and revised the manuscript. HY and MY participated in analysis of the data and revised the manuscript. All authors read and approved the final manuscript.

\section{Author details}

1 Department of General Medicine, Juntendo University School of Medicine, Hongo 2-1-1, Bunkyo-ku, Tokyo 113-8421, Japan. ${ }^{2}$ Department of Public Health, Juntendo University School of Medicine, Hongo 2-1-1, Bunkyo-ku, Tokyo 113-8421, Japan. ${ }^{3}$ Division of Health Science Research, Fukushima Welfare Federation of Agricultural Cooperatives, Sakamizu 50, Aizubange Town, Fukushima 969-6593, Japan. ${ }^{4}$ Department of Tumor and Host Bioscience, Fukushima Medical University School of Medicine, Hikarigaoka 1, Fukushima 960-1295, Japan.

\section{Acknowledgements}

We thank the participants of the study and the staff at Bange Kosei General Hospital and Takada Kosei Hospital for their assistance in data collection. This study was funded by a 2012 Grant-in-Aid for Scientific Research (C) (No. 24590816).

\section{Competing interests}

The authors declare that they have no competing interests.

Received: 16 October 2015 Accepted: 8 March 2016

Published online: 24 March 2016

\section{References}

1. Lozano R, Naghavi M, Foreman K, Lim S, Shibuya K, Aboyans V, et al. Global and regional mortality from 235 causes of death for 20 age groups in 1990 and 2010: a systematic analysis for the Global Burden of Disease Study. Lancet. 2012;380:2095-128.

2. Lakka HM, Laaksonen DE, Lakka TA, Niskanen LK, Kumpusalo E, Tuomilehto J, et al. The metabolic syndrome and total and cardiovascular disease mortality in middle-aged men. JAMA. 2002;288:2709-16.

3. Zavaroni I, Bonini L, Gasparini P, Barilli AL, Zuccarelli A, Dall'Aglio E, et al. Hyperinsulinemia in a normal population as a predictor of non-insulindependent diabetes mellitus, hypertension, and coronary heart disease: the Barilla factory revisited. Metabolism. 1999;48:989-94.

4. International Diabetes Federation. IDF worldwide definition of the metabolic syndrome. 2006. http://www.idf.org/webdata/docs/IDF_Meta_def_ final.pdf. Accessed 17 Aug 2015.

5. Ministry of Health, Labour and Welfare. National Health and Nutrition Examination Survey. 2008. http://www.mhlw.go.jp/houdou/2008/12/ h1225-5.html. (in Japanese) Accessed 17 Aug 2015.

6. Malik S, Wong ND, Franklin SS, Kamath TV, L'Italien GJ, Pio JR, et al. Impact of the metabolic syndrome on mortality from coronary heart disease, cardiovascular disease, and all causes in United States adults. Circulation. 2004;110:1245-50.

7. Yates KF, Sweat V, Yau PL, Turchiano MM, Convit A. Impact of metabolic syndrome on cognition and brain: a selected review of the literature. Arterioscler Thromb Vasc Biol. 2012;32:2060-7.

8. Umpierre D, Ribeiro PA, Kramer CK, Leitão CB, Zucatti AT, Azevedo MJ, et al. Physical activity advice only or structured exercise training and association with $\mathrm{HbA} 1 \mathrm{c}$ levels in type 2 diabetes: a systematic review and meta-analysis. JAMA. 2011;30:1790-9.

9. Rutten LJ, Arora NK, Bakos AD, Aziz N, Rowland J. Information needs and sources of information among cancer patients: a systematic review of research (1980-2003). Patient Educ Couns. 2005;57:250-61.

10. Hesse BW, Nelson DE, Kreps GL, Croyle RT, Arora NK, Rimer BK, et al. Trust and sources of health information: the impact of the Internet and its implications for health care providers: findings from the first Health Information National Trends Survey. Arch Intern Med. 2005;165:2618-24. 
11. Koch-Weser S, Bradshaw YS, Gualtieri L, Gallagher SS. The Internet as a health information source: findings from the 2007 Health Information National Trends Survey and implications for health communication. J Health Commun. 2010;15:279-93.

12. Powers BJ, Trinh JV, Bosworth HB. Can this patient read and understand written health information? JAMA. 2010;304:76-84.

13. Nutbeam D. Health literacy as a public health goal: a challenge for contemporary health education and communication strategies into the $21 \mathrm{st}$ century. Health Promot Int. 2000;15:259-67.

14. Shibuya A, Inoue R, Ohkubo T, Takeda Y, Teshima T, Imai Y, et al. The relation between health literacy, hypertension knowledge, and blood pressure among middle-aged Japanese adults. Blood Press Monit. 2011;16:224-30.

15. Ishikawa H, Nomura K, Sato M, Yano E. Developing a measure of communicative and critical health literacy: a pilot study of Japanese office workers. Health Promot Int. 2008;23:269-74.

16. Suka M, Odajima T, Kasai M, Igarashi A, Ishikawa $H$, Kusama M, et al. The 14-item health literacy scale for Japanese adults (HLS-14). Environ Health Prev Med. 2013;18:407-15.

17. Yokokawa H, Yuasa M, Sanada H, Hisaoka T, Fukuda H. Age- and sexspecific impact of health literacy on healthy lifestyle characteristics among Japanese residents in a rural community. Health. 2015;7:679-88.

18. Warnick GR, Knopp RH, FitzpatrickV, Branson L. Estimating low-density lipoprotein cholesterol by the Friedewald equation is adequate for classifying patients on the basis of nationally recommended cut points. Clin Chem. 1990;36:15-9.

19. Grundy SM, Cleeman Jl, Merz CN, Brewer HB Jr, Clark LT, Hunninghake DB, et al. National Heart, Lung, and Blood Institute; American College of Cardiology Foundation; American Heart Association. Implications of recent clinical trials for the National Cholesterol Education Program Adult Treatment Panel III guidelines. Circulation. 2004;110:227-39.

20. Belloc NB, Breslow L. Relationship of physical health status and health practices. Prev Med. 1972;1:409-21.

21. Yokokawa H, Goto A, Sanada H, Watanabe T, Felder RA, Jose PA, et al. Achievement status toward goal blood pressure levels and healthy lifestyles among Japanese hypertensive patients; cross-sectional survey results from Fukushima Research of Hypertension (FRESH). Intern Med. 2011;50:1149-56.
22. Ministry of Health, Labor and Welfare \& Ministry of Education, Culture, Sports, Science and Technology. Ethical Guideline for Epidemiological Studies. 2013. http://www.mhlw.go.jp/general/seido/kousei/i-kenkyu/ ekigaku/0504sisin.html. (in Japanese) Accessed 17 Aug 2015.

23. Shah LC, West P, Bremmeyr K, Savoy-Moore RT. Health literacy instrument in family medicine: the "newest vital sign" ease of use and correlates. J Am Board Fam Med. 2010;23:195-203.

24. Lassetter JH, Clark L, Morgan SE, Brown LB, VanServellen G, Duncan K, et al. Health literacy and obesity among native Hawaiian and Pacific Islanders in the United States. Public Health Nurs. 2015;32:15-23.

25. Chari R, Warsh J, Ketterer T, Hossain J, Sharif I. Association between health literacy and child and adolescent obesity. Patient Educ Couns. 2014;94:61-6.

26. Sharif I, Blank AE. Relationship between child health literacy and body mass index in overweight children. Patient Educ Couns. 2010;79:43-8.

27. Mancia G, Bombelli M, Facchetti R, Casati A, Ronchi I, Quarti-Trevano F, et al. Impact of different definitions of the metabolic syndrome on the prevalence of organ damage, cardiometabolic risk and cardiovascular events. J Hypertens. 2010;28:999-1006.

28. Davey J, Holden CA, Smith BJ. The correlates of chronic disease-related health literacy and its components among men: a systematic review. BMC Public Health. 2015;15:589.

29. Suka M, Odajima T, Okamoto M, Sumitani M, Igarashi A, Ishikawa $H$, et al. Relationship between health literacy, health information access, health behavior, and health status in Japanese people. Patient Educ Couns. 2015;98:660-8.

30. Osborn CY, Paasche-Orlow MK, Bailey SC, Wolf MS. The mechanisms linking health literacy to behavior and health status. Am J Health Behav. 2011;35:118-28.

31. Heijmans M, Waverijn G, Rademakers J, van der Vaart R, Rijken M. Functional, communicative and critical health literacy of chronic disease patients and their importance for self-management. Patient Educ Couns. 2015;98:41-8.

32. Inoue M, Takahashi M, Kai I. Impact of communicative and critical health literacy on understanding of diabetes care and self-efficacy in diabetes management: a cross-sectional study of primary care in Japan. BMC Fam Pract. 2013;14:40.

\section{Submit your next manuscript to BioMed Central and we will help you at every step:}

- We accept pre-submission inquiries

- Our selector tool helps you to find the most relevant journal

- We provide round the clock customer support

- Convenient online submission

- Thorough peer review

- Inclusion in PubMed and all major indexing services

- Maximum visibility for your research

Submit your manuscript at www.biomedcentral.com/submit
( Biomed Central 